\title{
Geschichte des Winterthurer Spitals
}

\author{
Von Adolf M. Fehr
}

Als erstes Spital ist in den mittelalterlichen Urkunden das Sondersiechenhaus zu St. Georgen am 24. Mai 1287 aufgeführt. Es diente ausschließlich zur Unterbringung von Aussätzigen.

Das Armen- und Krankenspital, dessen Gründungszeit unbekannt ist, finden wir erstmals 1306 als Stiftung der Bürgerschaft erwähnt. Der damaligen Zeit entsprechend fanden hier nicht nur Kranke, sondern auch unbemittelte Pfründer, Waisenkinder, Wanderburschen und Bettler Unterkunft. Letztere waren offenbar eine Landplage, die so rasch wie möglich «guetlich hinweg geferget» werden sollten. Abgesehen von kleinen Veränderungen genügte diese Mehrzweckanstalt am Neumarkt über 500 Jahre den Anforderungen der Bürgerschaft.

Zu Beginn des 19. Jahrhunderts erfolgten größere Erweiterungsbauten am gleichen Standort. Das Spital wurde nun als Bürgerliche Pfrund-, Armenund Krankenanstalt bezeichnet.

Pro Jahr wurden nun anfänglich ca. 160-190 Patienten, später bis zu 300 jährlich, gepflegt; davon waren 20-25\% Ausländer. Große Zuwanderungen infolge der Industrialisierung hatten zur Folge, daß nur noch $11 \%$ der ca. 10000 Einwohner eigentliche Stadtbürger waren. Es ist einleuchtend, daß diese Minderheit die Lasten des Spitals nicht mehr tragen konnte.

Es wurde daher 1869 beschlossen, die Abtrennung vom Bürgerlichen Armenwesen und die Übernahme durch die politische Gemeinde vorzunehmen. Nun machte man sich daran, einen Neubau zu planen. Vorerst war die Finanzierung dieses Einwohnerspitals abzuklären. Hier kam die Schenkung eines hochherzigen Stadtbürgers, Herrn Friedrich Imhoof-Hotze, in der Höhe von 100000 Franken zu Hilfe und gab den Anstoß für weitere Spenden von Firmen - vor allem Sulzer und Rieter - und von Privaten. Fast die Hälfte des Voranschlages wurde damit sichergestellt. Die Gesamtsumme für den Neubau betrug etwas weniger als 600000 Franken. Eine Subvention vom Kanton war nicht erhältlich; lediglich ein Beitrag von 50 Rappen später 75 Rappen - pro Pflegetag wurde zugesichert. Als neuer Standort wurde die Gegend am südwestlichen Fuße des Lindberges gewählt.

Nach diesen Vorarbeiten konnte im Mai 1874 der erste Spatenstich erfolgen. $2 \frac{1}{2}$ Jahre später, am 15. November 1876, wurde das Einwohnerspi- 
tal mit 90 Betten dem Betrieb übergeben. An diesem Tag traten 20 Patienten aus den früheren Räumlichkeiten am Neumarkt mit dem gesamten Personal in den Neubau über - insgesamt 18 Personen: Verwalter, Ärzte, Wärter und Wärterinnen und das Hilfspersonal.

Das neue Akutspital wurde gerade zur rechten Zeit bezogen, denn die Medizin machte schon in den nächsten Jahren ungeahnte Fortschritte. Denken wir nur an die Entdeckung der Bakterien, an die Anti- und Asepsis und die Röntgenstrahlen. Es verging jeweils nur eine kurze Zeit, bis im neuen Akutspital diese Entdeckungen den Kranken zugute kamen. Dazu einige Beispiele aus den Krankengeschichten, die fast vollständig erhalten sind. Sie sind teilweise gut und sorgfältig geführt, manchmal mit schönen Zeichnungen. Andere Krankengeschichten sind arg lückenhaft; Verlauf und Abschluß fehlen. So kann man heute noch, nach mehr als 100 Jahren, Rückschlüsse auf die Gewissenhaftigkeit der einzelnen Verfasser ziehen!

Unter den Krankheiten finden wir in erster Linie die Tuberkulose (Lungen, Gelenke und Knochen), die Diphtherie, die besonders bei den Kindern oft zum Tode an Erstickung führte. Zahlreich waren akute und chronische Rheumafälle sowie der Typhus. Einige Bluterkrankungen waren bekannt, wie zum Beispiel die perniziöse Anämie, die essentielle Thrombopenie, der Hodgkin, Bleivergiftung und anderes mehr.

Auch der chronische Alkoholismus war nicht selten: das Delirium potatorum furibundum, das mit Chloralhydrat, Zwangsjacke und Tobzelle behandelt wurde. Hierzu ein Beispiel aus dem Jahre 1877; ich zitiere wörtlich: «Der Patient ist ein alter Mann (61 Jahre), welcher im Verfluß eines tätigen Lebens (Kuhhirt) den Tod, die Flasche in der Hand, erwartet. Seine Nase verrät seine Leidenschaft durch ausgedehnte Gefäße.» - Man hat ihm offenbar die Flasche nicht weggenommen. Es fand sich auch keine Stadtbehörde, die den Chefarzt wegen passiver Euthanasie verzeigte!

An Medikamenten fällt besonders das Pilocarpin auf, das zu Schwitzkuren bei Ödemen und Exsudaten verwendet wurde, und das Curare bei Tetanus (Starrkrampf). Zwei Fälle sind erwähnt, von denen einer gerettet werden konnte. Es wurde fünf bis sechs Jahrzehnte später in USA wiederentdeckt.

Auf dem Gebiet der Chirurgie war wohl die Äther-Chloroform-Narkose längst bekannt, nicht aber die Ursache der Infektion. Man verwendete das Carbol nach Lister als Spray oder Kompressen zur Bekämpfung der in der Luft schwebenden «Miasmen», die sogenannte Antisepsis. Das Carbol führte nicht selten zu Vergiftungen und wurde daher 1887 durch Subli- 
mat ersetzt. Die Entdeckung der Bakterien führte dann bald zur Asepsis.

Wenn auch die septische Chirurgie im Vordergrund stand, wagten sich die Ärzte doch an größere Operationen: Strumektomie, Rektumamputation (nach Kraske), Oberkieferresektion, große Amputationen, besonders nach offenen Frakturen. Hauttransplantationen wurden nicht selten zur Dekkung großer Defekte vorgenommen.

Im alten Spital am Neumarkt wurde selten operiert, zirka zehn- bis zwanzigmal pro Jahr, im neuen Spital hingegen fünfmal häufiger oder mehr, da nun ein richtiger Operationssaal zur Verfügung stand.

Nur wenige Jahre nach der Eröffnung des Einwohnerspitals wurden von den Gemeinden der benachbarten Bezirke Andelfingen, Bülach und Pfäffikon Gesuche an den Kantonsrat eingereicht, wonach es den Kranken ihrer Region ermöglicht werden sollte, in Winterthur hospitalisiert zu werden. Denn das Einwohnerspital war ja im Grunde genommen nur den Stadtbewohnern zugänglich, wenn auch Ausnahmen gemacht wurden. Das heißt mit anderen Worten: Übernahme durch den Kanton.

Der Gedanke, das - wie es hieß - «Kleinod im Kranze der städtischen Anlagen» preiszugeben, kostete die Winterthurer eine schmerzliche Überwindung. Wir dürfen von Glück sagen, daß dieses Kleinod nicht unter Denkmalschutz gestellt wurde; sonst wären die späteren Neubauten vielleicht in Frage gestellt worden.

Doch gaben schließlich finanzielle Überlegungen den Ausschlag, das Einwohnerspital dem Kanton abzutreten. Durch den Konkurs der Nationalbahn war die Stadt in Schulden geraten, die durch den Verkauf wenigstens teilweise getilgt werden konnten. Nach längeren Verhandlungen einigte man sich auf einen Kaufpreis von 400000 Franken. Der Entschluß des Regierungsrates wurde dadurch erleichtert, daß wiederum ein Mäzen sich bereit erklärt hatte, dem Kanton 200000 Franken zum Zwecke der Übernahme des Einwohnerspitals zur Verfügung zu stellen. Es war der Winterthurer Industrielle J.Schoch in Mailand. Am 1. Januar 1886 wurde die Anstalt mit dem gesamten Personal vom Staat übernommen und hieß fortan Kantonsspital.

Es entbehrt nicht einer gewissen Ironie, daß die Zürcher Medizinische Fakultät sich schon zu Beginn der Verhandlungen vehement dagegen ausgesprochen hatte. Das Spital sollte als Asyl, Refugium für Rekonvaleszenten, Gebrechliche und chronische Leiden verwendet werden: die Schaffung eines Konkurrenzunternehmens gefährde die Medizinische Fakultät und sei gleichbedeutend mit dem Ruin der Hochschule. Als sie damit kein 
Gehör fand, wurde ein Expertenbericht, unterzeichnet von vier Professoren (Eichhorst, Krönlein, Horner und $\mathrm{W}_{\mathrm{y}} \beta$ ) eingereicht, worin eine Filiale des Zürcher Kantonsspitals vorgeschlagen wurde, in welche Akut- und Chronischkranke - aus der weiteren Umgebung von Zürich nur im Einverständnis ihres eigenen Aufnahmebüros - hospitalisiert werden durften.

Es ist dem damaligen Regierungsrat hoch anzurechnen, daß er dem Druck der hochwohllöblichen Professoren nicht nachgegeben hat; sonst wäre das Spital zu einer bloßen Versorgungs- oder Verpflegungsanstalt degradiert worden. Trotzdem ist die Zürcher Universität nicht eingegangen!

Wenn man bedenkt, daß das neue Kantonsspital den Staat nur 200000 Franken gekostet hat, d.h. zirka ein Drittel der Erstellungskosten, so versteht man, daß das Zürcher Volk mit großem Mehr von $45000 \mathrm{Ja}$ gegen 9000 Nein der Übernahme zugestimmt hat.

Das große neue Einzugsgebiet und die rasch wachsende Bevölkerungszahl führten schon bald zu einem Platzmangel an Betten, Laboratorien und im 1900 eingerichteten Röntgenkabinett. Um diesem ständigen Mangel abzuhelfen, wurden stückweise Erweiterungen vorgenommen, die jeweils nur kurze Zeit genügten. Folgende Bauten wurden erstellt, die nicht zuletzt durch die medizinischen Fortschritte im Laufe der folgenden Jahrzehnte bedingt wurden:

1895/97: Einstöckiges Absonderungshaus und Diphtheriegebäude.

1911: $\quad$ Poliklinik mit einem Parkplatz für Kinderwagen. Dieser Bau wird noch heute benützt, allerdings weniger für Kinderwagen. 1915/16: Aufstockung des Absonderungshauses und des Diphtheriegebäudes als intern-medizinische und geburtshilfliche Abteilung (der Lift wurde erst 1924 eingebaut).

Dies war die erste Spezialisierung, die schon lange vom Chefarzt Stierlin gewünscht, heute würde man sagen gefordert worden war.

Mit den drei selbständigen Abteilungen wurde 1917 der Prozeß der Spezialisierung eingeleitet; heute sind es total zwölf selbständige Kliniken und Institute.

1925: Aufstockung des Hauptgebäudes um zwei Stockwerke.

1937: Anbau an der geburtshilflichen Abteilung: Neue Operationsund Gebärsäle.

Seit1914 wurden für diese Erweiterungen 5,5 Millionen Franken ausgegeben. Die Bettenzahl stieg auf zirka 400, die Zahl der aufgenommenen Patienten auf 4000 . 
1940/41: Damals begann die Planung für eine großzügige Lösung des Spitalproblems. Mehrfach wurden die Pläne abgeändert, bis das Projekt reif war für die Volksabstimmung vom November 1947. Mit 112000 Ja gegen 58000 Nein wurde jede Erwartung übertroffen.

Da der Spitalbetrieb nicht unterbrochen werden durfte, konnte der Bau nur etappenweise vor sich gehen.

1950: Schwesternhaus an der Brunngasse, anschließend Ökonomiegebäude: Küche, Heizung, Werkstätten, Wäscherei.

1954/58: Neunstöckiges Bettenhaus und Behandlungstrakt als 1.Bauetappe, für Medizin, Chirurgie, Laboratorien, Röntgeninstitut und Pathologie.

1961: Abbruch des Hauptgebäudes - des «Kleinods».

1968: Eröffnung des Personalhauses und der Krankenpflegeschule an der Albanistraße.

Diese Schule, 1948 gegründet, hat maßgeblich dazu beigetragen, daß der Schwestern- und Pflegermangel weniger schwerwiegend war als an anderen Orten.

Noch im gleichen Jahr, 1968, konnte das fünfzehnstöckige Hochhaus für die Frauen-, Augen- und Kinderklinik dem Betrieb übergeben werden, als 2. Bauetappe.

1973: Eröffnung des neuen Polikliniktraktes, der anstelle der alten Frauenklinik errichtet wurde, als 3. Bauetappe.

Das heutige Zentralspital umfaßt nun zwölf Kliniken und Institute mit insgesamt 714 Betten. Es sind dies die drei Hauptkliniken Medizin, Chirurgie, Frauenklinik, die Medizinische Poliklinik, die Augen- und die Kinderklinik, die Rheumaklinik mit Physikalischer Therapie, die Psychiatrische Poliklinik, das Radiologische Institut, die Anästhesieabteilung, das Pathologische Institut und das Medizinisch-Chemische Zentrallaboratorium.

Wenn heute von gewisser Seite behauptet wird, daß im Spitalbau allgemein zu großzügig verfahren wurde und überdimensionierte Krankenhäuser erstellt wurden, so sind daran, zum mindesten teilweise, die Futurologen mit ihren falschen Bevölkerungsprognosen beteiligt. Wir dürfen beruhigt sein, daß dies in Winterthur in keiner Weise zutrifft. 
Die Geschichte des Spitals ist nicht nur als Rückblick auf vergangene Zeiten zu verstehen, sondern stellt uns zudem vor die Frage, wie sich die Zukunft gestalten wird. An Problemen, die in den kommenden Jahren an uns herantreten werden, mangelt es wahrlich nicht. Nur einige Punkte seien aus ärztlicher Sicht erwähnt:

1. Die rasch wachsenden Studentenzahlen lassen schon in wenigen Jahren eine Ärzte-Plethora erwarten.

2. Bessere Vorbereitung an den Hochschulen für die spätere Tätigkeit als praktische Ärzte, die jetzt durch die überspitzte Spezialisierung in den Schatten gestellt wird.

3. Die Spezialisierung hat mehr und mehr dazu geführt, daß die Krankheit in den Vordergrund gestellt wird, wobei man vergißt, die Kommunikation, das persönliche Gespräch mit dem kranken Menschen als Individuum, zu pflegen.

4. Die weltweite Kostenexplosion im Gesundheitswesen muß zwangsläufig zu gewissen Einschränkungen führen. Das Reizwort heißt: Optimal- statt Maximalmedizin. Kampf gegen Perfektionismus, zu dem wir Schweizer besonders neigen. Vermeidung von Doppelspurigkeiten. Gezielte, nicht schablonenhafte Untersuchung und Behandlung.

5. Um dies durchzusetzen, braucht es Autorität und Disziplin auf allen Stufen, wenn auch so etwas heute gar nicht beliebt îst.

6. Der Trend zur Verstaatlichung der Medizin, wie zum Beispiel in Schweden und England, gar nicht zu sprechen von den Ostblockländern, führt einerseits zu einer gewaltigen Kostensteigerung - so gibt es in England 30\% mehr Gesundheitsfunktionäre als Ärzte -; anderseits wird die Humanität, die Menschlichkeit, zugunsten des Kollektivismus zum Schwinden gebracht.

Dies sind einige Bemerkungen an die kommende Generation, die sich mit der Lösung dieser Probleme zu befassen haben wird.

Rückblickend gebührt unser Dank den einsichtigen Behörden von Stadt und Kanton, die sich für das Winterthurer Spitalwesen eh und je eingesetzt haben. Vor allem müssen wir dem Zürcher Volk danken, das all die Jahre hindurch den Spitalbauten großes Verständnis entgegengebracht hat. Schließlich sind wir zu Dank verpflichtet allen Mitarbeitern und Mitarbeiterinnen, die durch Jahre und Jahrzehnte hindurch mit Hingabe und Aufopferung dem kranken Menschen gedient haben. Das Beispiel der Alten zeigt den Weg in die Zukunft! 
Erinnern wir uns der Worte, die Billroth, der berühmte Chirurg in Zürich und Wien, 1870 geschrieben hat: «Es gibt nichts, was mehr vor Überhebung unserer Leistung schützt, als wenn man sich immer im Rahmen des Ganzen denkt.»

\section{Literatur}

100 Jahre Einwohner- und Kantonsspital Winterthur 1876-1976 von A. M. Fehr; zu beziehen beim Sekretariat der Verwaltungsdirektion des Kantonsspitals Winterthur, Tel. 052/864141, intern 212, zum Spezialpreis für Mitglieder der SGGMN von Fr. 10.-.

\section{Summary}

Winterthur, the second great town in the canton of Zurich, had already in 1287 a hospital for incurables, and in 1306 a hospital for poor and sick people. This has been enlarged in the beginning of the 19th century. In 1869 the poor were separated from the sick. A new hospital for the inhabitants opened its doors in 1876. Because the neighbour communities wanted to give their difficult cases also, the communal hospital changed into a cantonal hospital. The erection of new buildings was resolved in 1947. Twelve special clinics have been built in the 20 th century.

Prof. Dr.med. Adolf Max Fehr

Wylandstraße 33

CH-8400 Winterthur 